\title{
Canine breeds associated with gastric carcinoma, metaplasia and dysplasia diagnosed by histopathology of endoscopic biopsy samples
}

\author{
Marcus Vinicius Candido ${ }^{{ }^{*} \mathbb{D}}$, Pernilla Syrjä ${ }^{2}$, Susanne Kilpinen ${ }^{1}$ and Thomas Spillmann ${ }^{1}$
}

\begin{abstract}
Background: Gastric carcinoma (GC) is a rather rare pathological finding in dogs, with the exception of some breeds which seem predisposed. The etiopathogenesis is largely unknown in dogs, whereas in humans GC often develops from gastric mucosal metaplasia and dysplasia. This study investigates whether dogs of certain breeds are more often subject to gastroduodenoscopy (GDS), and diagnosed with GC, mucosal metaplasia or dysplasia. A retrospective clinical database search was performed at the Veterinary Teaching Hospital at the University of Helsinki, Finland. The following inclusion criteria were applied to estimate relative risk for metaplasia/dysplasia and GC: dogs from pure breeds with at least five individuals subject to GDS with histopathology of gastric biopsies.

Results: Between 2006 and 2016, from a total of 54945 canine patients presented, 423 dogs underwent GDS. Inclusion criteria were met in 180 dogs of 20 different pure breeds. Eight dogs had GCs (mean age $=9.8 \pm 1.7$ years): Belgian Tervuren $(n=4)$, Collie $(n=2)$, Golden Retriever $(n=1)$ and Jack Russel Terrier $(n=1)$. Fourteen dogs of eight breeds had gastric mucosal metaplasia or dysplasia. A log-binomial statistical model revealed that dogs in the following breeds had a significantly higher probability to undergo GDS than the others in the study population: Australian Terrier, Belgian Tervuren, Cairn Terrier, Collie and Siberian Husky. Belgian Tervuren was found at higher risk to be diagnosed with $G C[R R=19(5.7-63.9 ; P<0.0001)]$, as well as mucosal metaplasia/dysplasia [RR $(7.6 ; 2.95-19.58$; $P<0.0001)]$, as compared to the other breeds included. Shetland Sheepdog had an increased RR $(5.83 ; 1.75-19.45$; $P=0.0041$ ) for metaplasia.
\end{abstract}

Conclusions: The results indicate a very low incidence of GC in dogs. The Belgian Tervuren, however, appears as predisposed. The histopathologic descriptions of mucosal changes such as metaplasia and dysplasia were also rare, but were more frequent in the Belgian Tervuren. Previous reports of these changes in dogs are very scarce, but they might be presumably related to GC in dogs, as they are in humans. Future research should investigate the possible role of metaplasia and dysplasia in the development of GC in dogs, especially those of predisposed breeds.

Keywords: Breed predisposition, Cancer, Dog, Dysplasia, Endoscopy, Gastric carcinoma, Gastroduodenoscopy, Metaplasia

\footnotetext{
*Correspondence: marcus.candido@helsinki.fi

${ }^{1}$ Department of Equine and Small Animal Medicine, Faculty of Veterinary

Medicine, University of Helsinki, P.O. Box 57, 00014 Helsinki, Finland
}

Full list of author information is available at the end of the article 


\section{Background}

Gastric carcinoma (GC) is a rare pathologic finding in dogs, as it corresponds to less than one percent of all neoplastic changes identified in dogs $[1,2]$. The most common type of gastric neoplasm in dogs is adenocarcinoma, which is diagnosed at around 10 years of age, with initial clinical signs similar to those of other chronic gastrointestinal disorders [2-5]. Most gastrointestinal carcinomas in humans are known to evolve from superficial, flat changes classified as metaplastic or dysplastic according to their degree of cellular differentiation $[6,7]$. $\mathrm{GC}$ is the fifth most common type of cancer in people worldwide, and endoscopic biopsy is essential for diagnostic and staging [8]. Visual enhancement techniques such as chromoendoscopy and narrow band imaging are often applied for cancer screening and surveillance, as they can help improve the diagnostic yield of discrete mucosal changes [9]. The pathogenesis in humans is complex and not fully understood, but in approximately $90 \%$ of the cases it involves a multistep, multifactorial process. The progression from normal mucosa to chronic gastritis, further evolving to atrophic gastritis, intestinal metaplasia, dysplasia and adenocarcinoma, occurs over several years [10]. However, challenging this classic histogenetic pathway in which metaplasia and dysplasia are well-recognized pre-neoplastic changes, human GC can also develop from otherwise normal gastric mucosa. Diffuse gastric cancer, for example, is rare and often develops in a hereditary setting from foci of superficial signet ring carcinoma in situ [11]. Gastric infection with Helicobacter pylori plays a major role among environmental factors contributing to an increased risk of gastric cancer in humans [12], along with dietary factors including high intake of salt and preserved or smoked food (especially meat products), and low consumption of fresh fruit and vegetables [13].

The etiology of GC in dogs is still unknown, and it is suspected to be as complex as in humans [14]. The cardinal histological changes in canine gastric carcinogenesis remain undetermined, and the correlation between gastric polyps, Helicobacter spp. and GC has not yet been demonstrated $[5,15,16]$. A study on 14 Norwegian Lundehund dogs describes findings of mucous metaplasia in association with varying degrees of atrophic gastritis and intestinal lymphangiectasia. Additionally, four of those dogs had gastric neoplasia, which suggests that the changes represent a single pathological process [17]. In a further study with a total of eight Lundehunds with gastric neoplasia, it seems that such tumors are rather of neuroendocrine origin (derived from enterochromaffin-like-cells), possibly secondary to gastric atrophy and hypergastrinemia [18]. However, canine GC is not always associated with atrophic gastritis and comprehensive studies demonstrating the progression of atrophy towards metaplasia, dysplasia and gastric cancer in dogs are still lacking.

Previous retrospective studies based on data from canine cancer registry, records from specialists, pathology laboratories and breeders have shown breed predisposition to GC in the Belgian Tervuren, Bouvier des Flandres, Groenendael, Collie, Chow-Chow, Poodle, Norwegian Elkhound and a few other breeds, which implies a genetic background for the disease $[1,3,4,19$, 20]. In Finland, information about breed association to gastric cancer has been missing.

The present study was conducted to investigate which pure breeds are most commonly subject to gastroduodenoscopy (GDS) at the Veterinary Teaching Hospital of the University of Helsinki, a national referral center. The most important objective was to determine the probability of dogs in different breeds undergoing endoscopy with mucosal biopsy for histology to be diagnosed with gastric carcinoma, metaplasia or dysplasia, as a possible evidence of breed predisposition also in Finland. The overall aim of this study was to gather information about gastric cancer and metaplastic or dysplastic changes as recognizable features in canine patients undergoing endoscopy with gastric mucosal biopsy for histology. This study relies on the hypothesis that, in the clinical setting of an endoscopic unit, certain breeds can be found affected with GC and mucosal metaplasia/dysplasia more commonly than others. The study therefore aims at investigating a possible association between individual breed, submission to GDS, and diagnosis of gastric carcinoma and metaplastic or dysplastic mucosal changes in histopathology.

\section{Methods}

The study was designed as a retrospective cohort on the diagnosis of GC, metaplasia and dysplasia in dogs subjected to GDS. The source population of this study comprises all dogs presented at the Veterinary Teaching Hospital between January 2006 and December 2016. A computerized database search was performed on the records throughout this period. The study population included dogs which belong to a pure breed having a minimum of five dogs subject to GDS with complete histopathological report based on gastric biopsies. The Belgian Tervuren is herein regarded as a breed on its own. The breed Collie includes both rough and smooth haircoat varieties (Border Collie and Shetland Shepherd being separate pure breeds).

The endoscopic biopsy samples were collected in a standardized fashion: at least eight gastric mucosal fragments were taken onto wood chips and then immediately immersed (upside down) in 10\% neutral buffered formalin. After $24 \mathrm{~h}$ fixation, the biopsies were processed, 
paraffine-embedded, sectioned at 4 microns and stained with hematoxylin and eosin (HE). Special staining such as periodic acid-Schiff (PAS) or Warthin-Starry (WS) were applied when indicated.

The endoscopic mucosal biopsy samples were examined at the University of Helsinki by veterinary pathologists adhering to a standardized assessment protocol since 2006. The standard reporting template included the following parameters as main criteria for histopathological evaluation of the stomach: number of biopsy samples examined, epithelial surface characterization, amount of intraepithelial lymphocytes, presence of Helicobacter-like organisms, characterization and amount of mononuclear and granulocyte infiltration in lamina propria, presence of lymphoid follicles, characterization of fundic glands, and whether pepsinogen and $\mathrm{HCl}$-producing cells are identified regularly, or else substituted by mucous cells (metaplasia) [21]. Gland structure was also evaluated regarding presence of fibrosis, glandular nesting and structural changes which could be related to dysplasia. The World Small Animal Veterinary Association standards and guidelines were applied from 2008 onwards and the template was updated accordingly, regarding grading of inflammatory changes (0-3: normal, mild, moderate, severe) [22, 23]. The diagnoses were given under the supervision of, and in consensus with, a board-certified pathologist (PS).

The complete histopathological record of endoscopic biopsies from each patient of the study population was revisited for findings consistent with either gastric carcinoma (GC), dysplasia or metaplasia, in addition to other features [21-23]. Histologic sections from the patients presenting these specific lesions were re-examined by a board-certified pathologist (PS) for verification of the diagnosis and consistency of the results.

\section{Statistical methods}

All of the outcomes tested were binary, because the presence or absence of GDS, GC or metaplasia/dysplasia was considered for each case studied. A log-binomial model was applied for each outcome variable, and the probability of presence was modelled with $95 \%$ confidence intervals (CI). The models were re-run separately for each breed in the analysis population comparing the breed of interest to other breeds. Relative probability to undergo GDS (numerically equivalent to relative risk) was calculated for each selected breed in comparison to all other canine patients visiting the hospital in the period (source population). The individual histopathological diagnosis from dogs of breeds meeting the inclusion criteria (study population) was considered for the calculation of relative risk (RR) to be diagnosed with gastric metaplasia/ dysplasia, or carcinoma, and computed for each breed in comparison to the other breeds included. $P$-values $<0.05$ were considered significant. All analyses were done using SAS $^{\circledR}$ System for Windows, version 9.3 (SAS Institute Inc., Cary, NC, USA).

\section{Results}

Pure breeds undergoing gastroduodenoscopy (GDS)

Between 2006 and 2016, the source population included a grand total of 54,945 canine patients being presented at the hospital. The study population was recruited out of 423 dogs which underwent GDS for diagnostic reasons, 30 of which being mixed-breed dogs. The inclusion criteria mentioned above were met by 180 dogs representing 20 different pure breeds. The study population consisted of 75 intact males, 26 castrated males, 46 intact females

Table 1 Study population: breeds with at least five dogs undergoing gastroduodenoscopy (GDS) and histopathological examination of biopsy samples, selected for inclusion from the source population of all dogs presented between 01.01.2006 and 31.12.2016

\begin{tabular}{|c|c|c|}
\hline Breed & $\begin{array}{l}\text { Total dogs } \\
\text { presented }\end{array}$ & $\begin{array}{l}\text { Dogs } \\
\text { undergoing } \\
\text { GDS }\end{array}$ \\
\hline Australian Terrier & 245 & 5 \\
\hline Belgian Tervuren & 196 & 9 \\
\hline Border Collie & 430 & 5 \\
\hline Cairn Terrier & 522 & 11 \\
\hline Chihuahua & 1729 & 5 \\
\hline Collie & 673 & 27 \\
\hline Dachshund & 1609 & 7 \\
\hline French bulldog & 845 & 7 \\
\hline German Shepherd & 2110 & 18 \\
\hline Golden Retriever & 1216 & 15 \\
\hline Hovawart & 328 & 5 \\
\hline Jack Russell Terrier & 1149 & 7 \\
\hline Labrador retriever & 2124 & 14 \\
\hline Miniature Schnauzer & 1003 & 7 \\
\hline Parson Russell Terrier & 496 & 6 \\
\hline Rottweiler & 794 & 10 \\
\hline Shetland Sheepdog & 734 & 5 \\
\hline Siberian Husky & 219 & 5 \\
\hline Staffordshire Bull Terrier & 592 & 6 \\
\hline West Highland White Terrier & 690 & 6 \\
\hline Dogs in the breeds above & 17,704 & 180 \\
\hline Other breeds/mixed (not included) & 37,241 & $243^{a}$ \\
\hline All dogs in the period & 54,945 & $423^{b}$ \\
\hline
\end{tabular}

Study population is indicated in bolditalic

Source population is indicated in italic

a Includes individual dogs from the selected breeds which were excluded from the study due to lack of histopathological diagnosis (foreign body retrieval, no biopsy taken or biopsy lost to follow-up)

b Total number of GDS patients in the period 


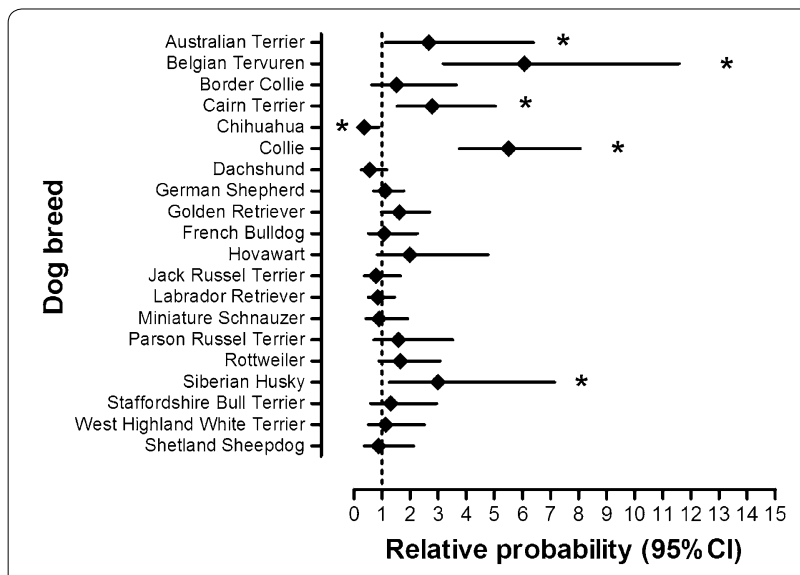

Fig. 1 Relative probability to undergo GDS for dogs of the selected breeds in comparison to all other dogs in the source population. *The difference is statistically significant $(P<0.05)$

and 33 sterilized females, which underwent GDS at the mean age of $5.3 \pm 3.4$ years $(n=180)$. These dogs correspond to $43 \%$ of all GDS patients in the period (Table 1 ). Twelve other individual dogs of the breeds included had already been excluded from the study either because histopathological information was lost $(n=3)$ or no biopsies were taken during GDS $(n=9)$. Six of those nine dogs had gastric foreign bodies removed (plastic; wood; needles; stone) and one presented biliary tract changes and underwent endoscopic retrograde cholangiopancreatography followed by surgical removal of the gallbladder.

When comparing the study population with the source population, a log-binomial model revealed significantly higher relative probability to undergo GDS in the following breeds as compared to dogs of other breeds (RR; $95 \%$ CI lower-higher; $P$ value): Australian Terrier (2.67; 1.126.39; $P=0.0274)$, Belgian Tervuren (6.07; 3.18-11.58; $P<0.0001)$, Cairn Terrier $(2.78 ; 1.54-5.03 ; P=0.0007)$, Collie $(5.5 ; 3.75-8.06 ; P<0.0001)$ and Siberian Husky (2.99; $1.25-7.15 ; P=0.0138)$. Chihuahuas underwent GDS significantly less often than dogs of other breeds (0.37; 0.15-0.89; $P=0.0261$ ) (Fig. 1).

\section{Gastric carcinoma}

Eight of the dogs included in the study were diagnosed with gastric adenocarcinomas, which was the only type of gastric neoplasm seen in the study population. Affected dogs belonged to the following breeds: Belgian Tervuren $(\mathrm{n}=4$, including one signet-cell carcinoma), Collie $(n=2)$, Golden Retriever and Jack Russel Terrier (Table 2). Five of them were female and three were male. Concerning age at diagnosis of GC, the youngest patient was 7.8 years old when the examination was performed (mean age $=9.8 \pm 1.7$ years; see also Table 3 ). The Belgian
Table 2 Number of dogs in each breed undergoing gastroduodenoscopy (GDS), and diagnosed with gastric carcinoma (GC), dysplasia or metaplasia from endoscopic biopsy samples taken from 01.01.2006 to 31.12.2016

\begin{tabular}{lrll}
\hline Breed & GDS & GC & $\begin{array}{l}\text { Dysplasia } \\
\text { or metaplasia }\end{array}$ \\
\hline Belgian Tervuren & 9 & 4 & 4 \\
Collie & 27 & 2 & 2 \\
Dachshund & 7 & - & 1 \\
Golden Retriever & 15 & 1 & 2 \\
Hovawart & 5 & - & 1 \\
Jack Russell Terrier & 7 & 1 & - \\
Shetland Sheepdog & 5 & - & 2 \\
Siberian Husky & 5 & - & 1 \\
Staffordshire Bull Terrier & 6 & - & 1 \\
Total in these breeds & 86 & 8 & 14 \\
\hline
\end{tabular}

Tervuren was found at much greater relative risk for being diagnosed with GC from histopathological examination of endoscopically obtained gastric biopsies (33.4; 6.43-173.36; $P<0.0001$ ) in comparison to other breeds (Fig. 2).

\section{Metaplasia and dysplasia in gastric mucosa}

Changes histologically described as metaplastic or dysplastic were seen in 14 dogs in the following breeds: Belgian Tervuren $(n=4)$, Collie $(n=2)$, Golden Retriever $(\mathrm{n}=2)$, Shetland Sheepdog $(\mathrm{n}=2)$, Dachshund $(\mathrm{n}=1)$, Hovawart $(n=1)$, Siberian Husky $(n=1)$ and Staffordshire Bull Terrier $(n=1)$. The mean age when these patients underwent GDS was $7.9 \pm 2.6$ years. Significant statistical differences were found for the diagnosis of either metaplasia or dysplasia in Belgian Tervuren compared to other breeds: RR $(7.6 ; 2.95-19.58 ; P<0.0001)$. The Shetland Sheepdog was found at significantly increased RR $(5.83 ; 1.75-19.45 ; P=0.0041)$ for metaplasia (dysplasia was not seen in this breed) compared to dogs of other breeds (Fig. 3).

\section{Endoscopic and histopathological diagnosis}

The final diagnosis considered for all of the analyses was achieved by endoscopic examination and sampling, followed by histopathological confirmation. All dogs presenting the changes of concern were also found with concomitant inflammatory changes (Table 3). Each set of sample images shown in this article correspond to a single patient, respectively diagnosed as carcinoma (Figs. 4, 5), metaplasia (Fig. 6) or dysplasia (Fig. 7). Chromoendoscopy and narrow band imaging were applied in one Belgian Tervuren presented with chronic vomiting, whose brother from the same litter had been euthanized 
Table 3 Breed, age (years), sex and concomitant changes found in each of the dogs diagnosed with gastric carcinoma (GC)

\begin{tabular}{|c|c|c|c|c|}
\hline Breed & Age & Sex & Inflammatory change & Structural change \\
\hline \multirow[t]{4}{*}{ Belgian Tervuren $(n=4)$} & 7.8 & $\mathrm{~F}$ & Moderate chronic LP G & Glandular dysplasia \\
\hline & 9.7 & $\mathrm{~F}$ & Moderate chronic LP G & - \\
\hline & 9.2 & M & $\begin{array}{l}\text { Moderate chronic LP G with neutrophilic com- } \\
\text { ponent and severe gland hyperplasia }\end{array}$ & $\begin{array}{l}\text { Glandular dysplasia, } \\
\text { epithelial dysplasia and } \\
\text { atypia }\end{array}$ \\
\hline & 11.0 & M & Severe chronic LP G & - \\
\hline \multirow[t]{2}{*}{ Collie $(n=2)$} & 8.6 & $\mathrm{~F}$ & Moderate chronic LP G & - \\
\hline & 8.5 & Fs & Moderate chronic atrophic $G$ & Mucinous metaplasia \\
\hline Golden Retriever & 10.8 & $\mathrm{~F}$ & $\begin{array}{l}\text { Severe chronic LP G with neutrophilic compo- } \\
\text { nent and ulcerative mycotic G }\end{array}$ & - \\
\hline Jack Russell Terrier & 12.9 & Mc & Severe gland hyperplasia, mild LPG & - \\
\hline
\end{tabular}

LP lymphoplasmacytic, G gastritis, Fs sterilized female, $M c$ castrated male

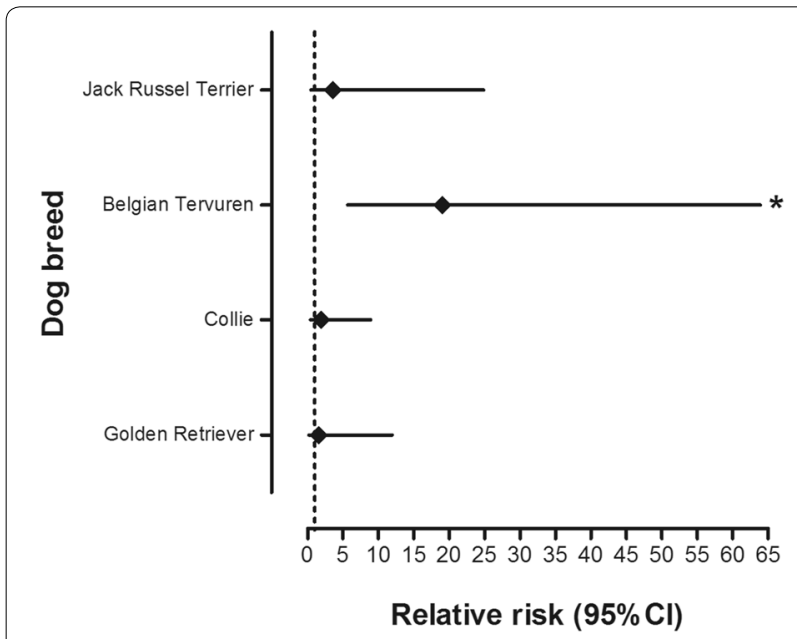

Fig. 2 Relative risk to be diagnosed with gastric carcinoma for selected breeds in comparison to the other breeds in the study population. ${ }^{*}$ The difference is statistically significant $(P<0.0001)$

due to a massive GC. The most prominent endoscopic and respective histopathological changes found in this patient are in Fig. 7.

\section{Discussion}

Some dog breeds (namely Australian Terrier, Belgian Tervuren, Cairn Terrier, Collie and Siberian Husky) underwent GDS significantly more often as compared to the other breeds in this study. Chihuahua was scoped significantly less often than others; this breed with a relatively small body size is rather common in the source population. These findings remain largely unexplained, however breed distribution (Table 1) and relatively low overall frequency of GDS (low sample sizes) may have influenced the results. It is not clear whether it was the severity of

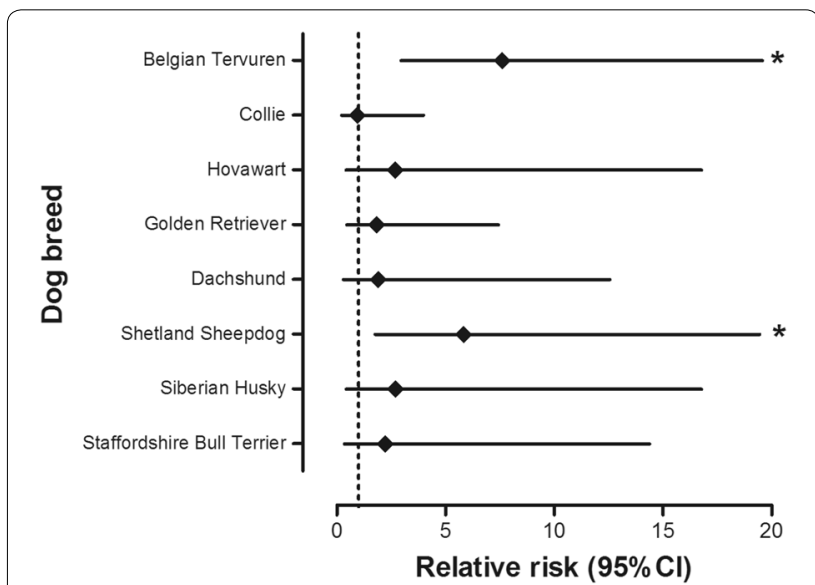

Fig. 3 Relative risk to be diagnosed with gastric mucosal metaplasia or dysplasia for selected breeds in comparison to the other breeds in the study population. *The difference is statistically significant $(P<0.005)$

gastrointestinal signs alone, or any other issues that might have influenced the probability for certain breeds to undergo GDS. It would be reasonable to speculate that an expected breed predisposition to $\mathrm{GC}$, as previously reported for the Belgian Tervuren [1, 3, 19], might potentially bias the approach of clinicians and owners towards submitting patients of this breed to endoscopy earlier, or more often. The fact that dogs of certain breeds undergo GDS more often might as well indicate a higher prevalence of gastrointestinal disorders in general, which may warrant more extensive studies in such breeds.

The Belgian Tervurens that underwent GDS in this study were consistently found at much higher risk of being diagnosed with GC than dogs of any other breed. This finding is in accordance with previous studies that used pathological records and cancer registry data of 

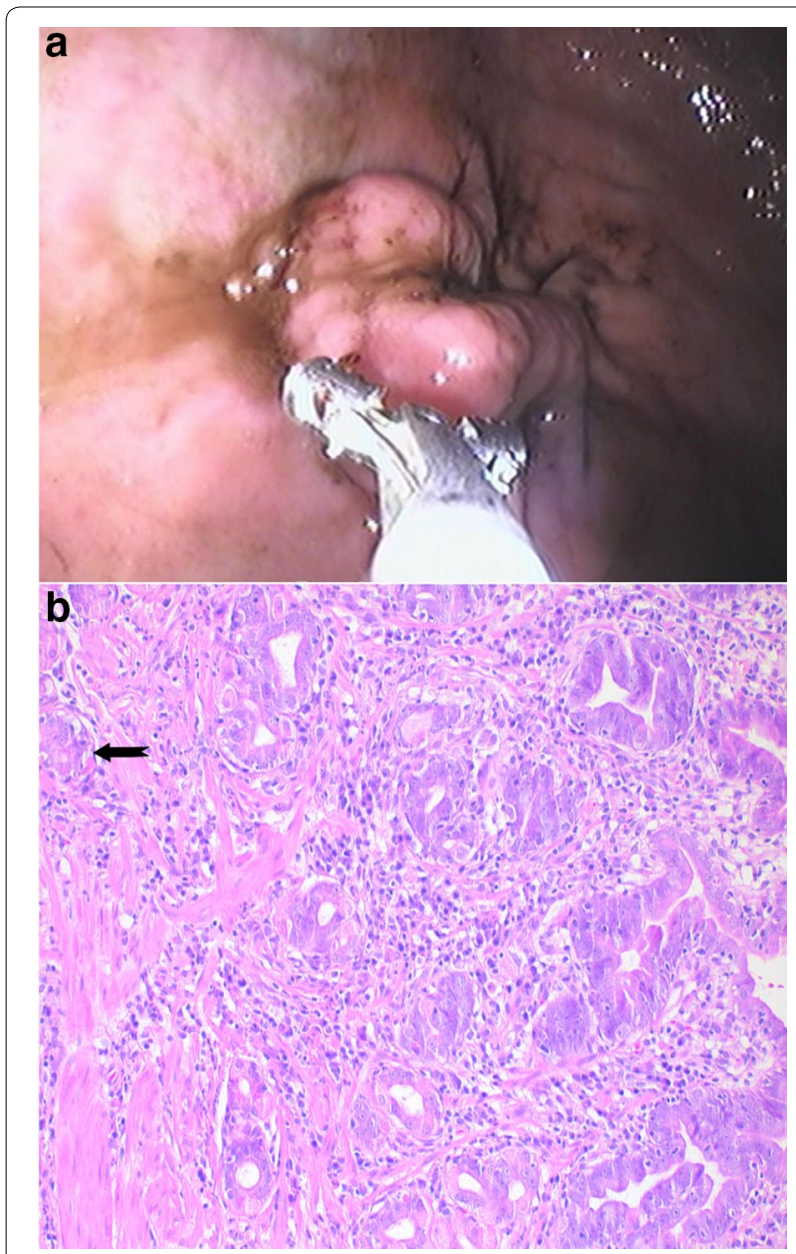

Fig. 4 Endoscopic and corresponding histologic images of an 8-year-old female Belgian Tervuren showing gastric adenocarcinoma. a White light endoscopic image of a gastric adenocarcinoma. Histology: b Well differentiated gastric adenocarcinoma with invasion of the muscularis mucosae (arrow). HE stain, Obj. 20x

other countries such as Italy, Netherlands or Norway, in which the Belgian Tervuren were also considered to be highly predisposed to GC $[1,3,19]$.

Gastric metaplasia or dysplasia were almost as rarely seen as GC, with significantly higher relative risks found for the Belgian Tervuren and Shetland Sheepdog. Although statistical significance could not be confirmed for every breed, both types of changes were found in Belgian Tervuren, Collie and Golden Retriever, sometimes in the same patient. Nonetheless, metaplasia and dysplasia can present as discrete, flat changes that are easily overlooked and possibly underdiagnosed [24], especially when considering the limitations of current white light endoscopy modalities available and non-directed sampling procedures in veterinary medicine. Visual enhancement techniques such as chromoendoscopy and narrow

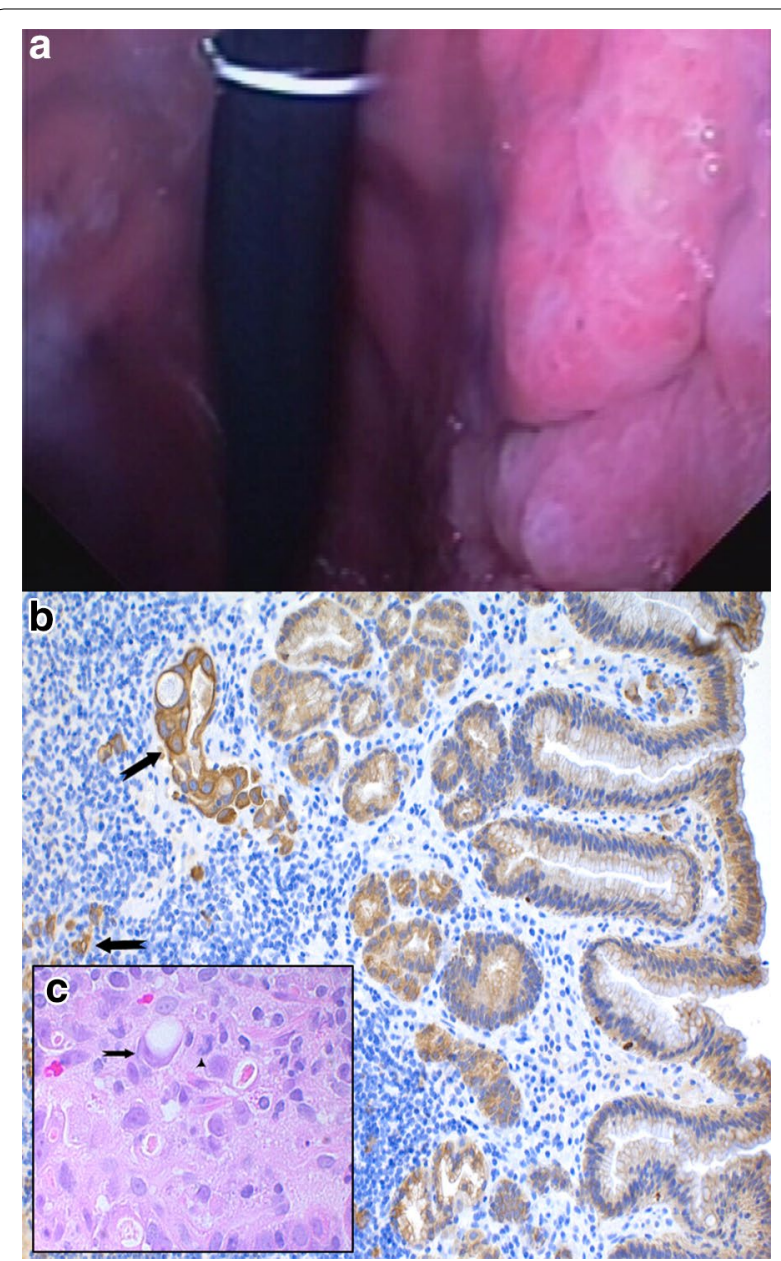

Fig. 5 Endoscopic and corresponding histologic images of a 10-year old female Belgian Tervuren representing gastric adenocarcinoma. a White light endoscopic image of a diffuse mass in the stomach. Histology: b Invasive gastric adenocarcinoma, with atypical tubular structures (arrow) and single cell invasion of deeper layers of the gastric wall (arrow), and c classical signet ring cells (arrow). b $\mathrm{HC}$ cytokeratin stain, Obj. 20x. c HE stain, Obj. 40x

band imaging (Fig. 7) may help improve the diagnostic yield of discrete mucosal changes [9].

One of the major challenges in veterinary gastroenterology is that the extension and severity of macroscopic changes seen during endoscopy are not always consistent with the respective histopathological findings from endoscopic biopsy [25]. Cognitive aspects including specific knowledge, endoscopist experience and training, standardized grading systems, image quality and meticulous examination techniques are fundamental for the detection of subtle lesions [26]. In this study, the biopsies and histologic sections were prepared and then examined according to a uniform in-house standard. Such protocols help to control the risk of inaccuracy related to lack 


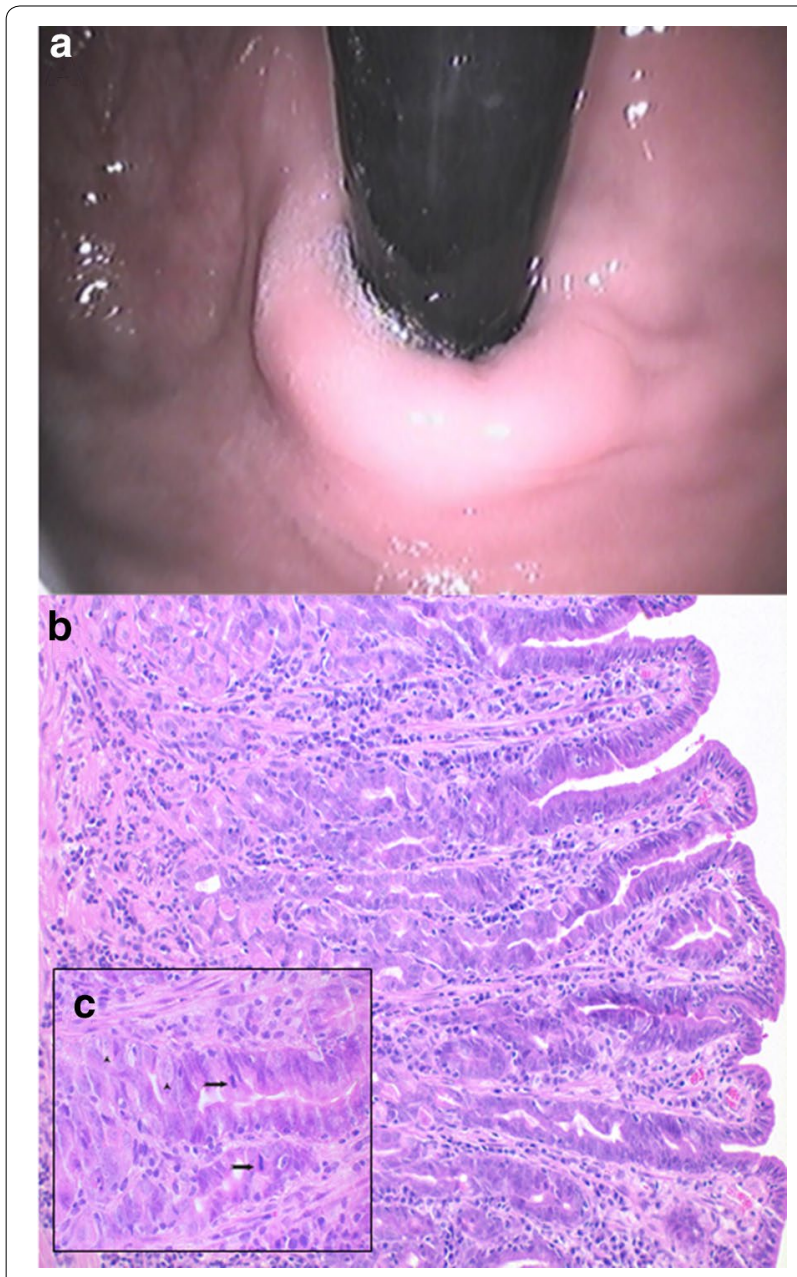

Fig. 6 Endoscopic and corresponding histologic images of a 9-year-old castrated male Dachshund representing metaplasia. a White light endoscopic image of gastric fundus with discrete mucosal changes. Histology: b Mucous/intestinal metaplasia within the gastric fundus; elongated hyperplastic mucous neck regions of fundic glands and diffuse moderate lymphoplasmacytic gastritis. c Increased mitotic figures within the hyperplastic mucous cells of the glandular neck area (arrow). Scattered parietal cells are visible (arrowhead) within the basal area of the glands. HE stain, b Obj. 20x c Obj. $40 x$

of uniformity on sample quality and to interobserver variation in histopathological evaluation. Such issues have been reported as potentially detrimental when histologic material or reports originated from several different institutions are utilized [27, 28].

The diagnosis of metaplastic or dysplastic changes in breeds with predispositions to GC might indicate a stepwise progression in dogs, comparable to the better known pathway towards GC in humans [10]. Studies in Lundehund dogs with GC ponder on a similar pathogenesis $[17,18]$. This might be the case especially for

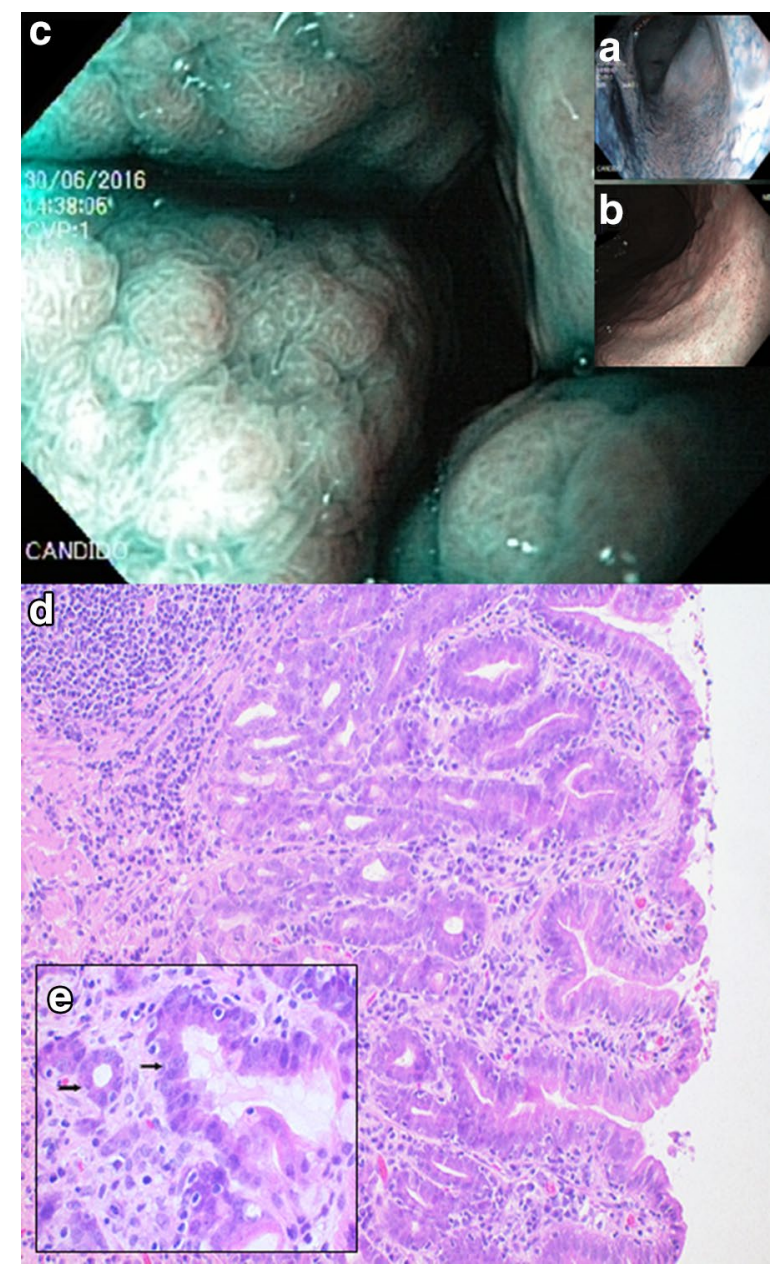

Fig. 7 Endoscopic and corresponding histologic images of a 9-year-old female Belgian Tervuren showing dysplasia. Endoscopic images $(\mathbf{a}, \mathbf{b}, \mathbf{c})$ show diffuse mucosal texture irregularity along gastric fundus and body: a chromoendoscopy (CE), b narrow band imaging (NBI), c CE and NBI combined. Histology: $\mathbf{d}$ dysplasia of the fundic glands, with glandular dilatation and distortion. e Cellular dysplasia with flattening of the glandular cells and loss of cellular polarity (arrows). HE stain, d Obj. 20x, e Obj. 40x

the Belgian Tervuren with its significantly higher relative risk for the suspected pre-neoplastic changes, along with its marked predisposition for GC. This hypothesis might be further explored by staging suspected lesions and screening dogs selected from predisposed breeds. Research should aim at applying more advanced endoscopic approaches such as chromoendoscopy or narrow band imaging, which are proven modalities in humans for improving the targeted sampling of lesions [29] that might be easily overlooked when using white light endoscopy alone. Metaplasia and dysplasia have not been consistently studied in canine gastroenterology and their potentially relevant role in the pathogenesis 
of canine GC must be further investigated, whereas in humans well-established staging protocols and advanced therapeutic approaches are routinely applied [30]. While standard guidelines for histopathology evaluation remain fluid in veterinary medicine, novel immune staining techniques and genetic examination of biopsy samples may provide valuable opportunities for both research and diagnosis in the near future [31].

The retrospective nature of the study and the sole inclusion of dogs that had undergone GDS led to some limitations that possibly influenced the interpretation of our results. This study did not include GC diagnosed after necropsy or full-thickness biopsy but rather focused on the findings of endoscopic biopsy samples. This approach resulted in a small case load, as endoscopy is typically performed in a relatively small proportion of dogs in the clinical population, thus it relies on specific indications and also the owner's compliance. A possible bias of indication for endoscopic procedures by different clinicians (e.g. awareness of certain breed predispositions), over such an extended time period, cannot be ruled out.

It is also worth mentioning that even in this selected population of canine patients with clinical signs of gastrointestinal disease, GCs were seen only in few individuals $(8 / 180=4.4 \%)$, which further corroborates the rarity of the condition. The frequency of disease in this study cannot be directly compared with previous studies that used different approaches. However, the low frequency of diagnosis and the advanced age at diagnosis of GC in dogs found in this setting are in agreement with previous reports $[1-4,14]$.

Gaining knowledge about the actual prevalence of metaplasia and dysplasia in dogs may help to determine their possible role as pre-neoplastic changes. Furthermore, patient follow-up after the diagnosis of metaplastic and dysplastic changes might contribute to the knowledge about canine gastric carcinogenesis. Multi-center, prospective studies that utilize standardized approaches could help overcome the limitations related to reduced sample sizes in veterinary research, especially with regard to uncommon disease conditions like the ones reported in this study.

\section{Conclusions}

Belgian Tervurens, Collies, Siberian Huskies, Cairn Terriers and Australian Terriers underwent GDS more often than dogs of other breeds in our study population. The Belgian Tervurens were found to be at much higher risk (19-fold) of being diagnosed with GC than dogs of other breeds, which further corroborates predisposition for GC in this breed. Metaplastic or dysplastic changes were slightly more frequent than GC. Although no causal relationship could be established thus far, the presence of lesions of all three types in breeds such as the Collie, the Golden Retriever, and chiefly the Belgian Tervuren, even in the face of these low case numbers, might be a sign of associated pathogenesis requiring further investigation.

\section{Authors' contributions}

MVC applied the inclusion criteria to the database, revised the histopathological record of the individual patients included, prepared the data for statistical analysis, selected and prepared images, tables and graphics, and was the main responsible for the manuscript. PS acted as a co-author and consultant in pathology, and provided the photomicrographs and respective histopathological highlights, also participating as an active reviewer when drafting the manuscript. SK performed part of the endoscopies and acted as co-supervisor involved in drafting the manuscript. TS was the research director. He performed part of the endoscopies, the baseline search in the hospital database, co-authored and reviewed the manuscript on all steps. All authors read and approved the final manuscript.

\section{Author details}

${ }^{1}$ Department of Equine and Small Animal Medicine, Faculty of Veterinary Medicine, University of Helsinki, P.O. Box 57, 00014 Helsinki, Finland. ${ }^{2}$ Department of Veterinary Biosciences, Faculty of Veterinary Medicine, University of Helsinki, P.O. Box 66, 00014 Helsinki, Finland.

\section{Acknowledgements}

Thanks go to Sofia Männikkö of '4Pharma' for her statistical analyses and technical assistance.

\section{Competing interests}

The authors declare that they have no competing interests.

\section{Availability of data and materials}

Most of the data generated or analysed during this study are included in this published article. Additional datasets used and/or analysed during the current study are available from the corresponding author on reasonable request.

\section{Consent for publication}

Not applicable. Histopathological and endoscopic images from canine patients do not disclose patient or owner identity.

Ethics approval and consent to participate

Not applicable. No sensitive data was recovered nor exposed.

\section{Funding}

This study was partly supported by a grant from the Brazilian Council for Science and Technology Development (CNPq). We also thank the Finnish Veterinary Foundation, the Finnish Foundation of Veterinary Research, and the Doctoral Program in Clinical Veterinary Medicine, for financial support.

\section{Publisher's Note}

Springer Nature remains neutral with regard to jurisdictional claims in published maps and institutional affiliations.

Received: 19 October 2017 Accepted: 8 June 2018

Published online: 18 June 2018

References

1. Seim-Wikse T, Jörundsson E, Nødtvedt A, Grotmol T, Bjornvad CR, Kristensen AT, et al. Breed predisposition to canine gastric carcinoma-a study based on the Norwegian canine cancer register. Acta Vet Scand. 2013;55:25.

2. Terragni R, Vignoli M, van Bree HJ, Gaschen L, Saunders JH. Diagnostic imaging and endoscopic finding in dogs and cats with gastric tumors: a review. Schweiz Arch Tierh. 2014;156:569-76. 
3. Lubbes D, Mandigers PJ, Heuven HC, Teske E. Incidence of gastric carcinoma in Dutch Tervueren shepherd dogs born between 1991 and 2002 Tijdschr Diergeneesk. 2009;134:606-10.

4. Von Babo V, Eberle N, Mischke R, Meyer-Lindenberg A, HewickerTrautwein M, Nolte I, et al. Canine non-hematopoietic gastric neoplasia. Tierarztl Prax Ausg K Kleintiere Heimtiere. 2012;40:243-9.

5. Taulescu MA, Valentine BA, Amorim I, Gärtner F, Dumitraşcu DL, Gal AF, et al. Histopathological features of canine spontaneous non-neoplastic gastric polyps-a retrospective study of 15 cases. Histol Histopathol. 2014;29:65-75.

6. Park YH, Kim N. Review of atrophic gastritis and intestinal metaplasia as a premalignant lesion of gastric cancer. J Cancer Prev. 2015;20:25.

7. Schlemper RJ, Riddell RH, Kato YE, Borchard F, Cooper HS, Dawsey SM, et al. The Vienna classification of gastrointestinal epithelial neoplasia. Gut. 2000;47:251-5.

8. Carneiro F. Stomach cancer. In: Stewart B, Wild CP, editors. International agency for research on cancer. Lyon: WHO; 2014. p. 383-91.

9. Trivedi PJ, Braden B. Indications, stains and techniques in chromoendoscopy. QJM. 2013;106:117-31.

10. Correa P. Human gastric carcinogenesis: a multistep and multifactorial process - first American Cancer Society award lecture on cancer epidemiology and prevention. Cancer Res. 1992;52:6735-40.

11. Carneiro F, Huntsman DG, Smyrk TC, Owen DA, Seruca R, Pharoah P, et al. Model of the early development of diffuse gastric cancer in E-cadherin mutation carriers and its implications for patient screening. J Pathol. 2004;203:681-7.

12. Forman D, Newell DG, Fullerton F, Yarnell JW, Stacey AR, Wald N, Sitas F. Association between infection with Helicobacter pylori and risk of gastric cancer: evidence from a prospective investigation. BMJ. 1991;302:1302-5.

13. World Cancer Research Fund/American Institute for Cancer Research. Food, nutrition, physical activity, and the prevention of cancer: a global perspective. Washington DC: AICR; 2007. p. 265-6.

14. Hugen S, Thomas RE, German AJ, Burgener IA, Mandigers PJ. Gastric carcinoma in canines and humans, a review. Vet Comp Oncol. 2017;15:692-705

15. Neiger R, Simpson KW. Helicobacter infection in dogs and cats: facts and fiction. J Vet Intern Med. 2000;14:125-33.

16. Amorim I, Taulescu MA, Ferreira A, Rêma A, Reis CA, Faustino AM, et al. An immunohistochemical study of canine spontaneous gastric polyps. Diagn Pathol. 2014;9:166.

17. Kolbjørnsen $\varnothing$, Press CM, Landsverk T. Gastropathies in the Lundehund. APMIS. 1994:102:647-61.

18. Qvigstad G, Kolbjørnsen $\varnothing$, Skancke E, Waldum HL. Gastric neuroendocrine carcinoma associated with atrophic gastritis in the Norwegian Lundehund. J Comp Pathol. 2008;139:194-201.
19. Scanziani E, Giusti AM, Gualtieri M, Fonda D. Gastric carcinoma in the Belgian shepherd dog. J Small Anim Pract. 1991;32:465-9.

20. Bilek A, Hirt RA. Breed-associated increased occurrence of gastric carcinoma in Chow-Chows. Wien Tierarztl Monatsschr. 2007;94:71-9.

21. Plattner B, Hostetter JM, Uzal FA. Alimentary system and peritoneum. In: Maxie MG, editor. Jubb, Kennedy and Palmer's pathology of domestic animals, vol. 2. St. Louis: Elsevier; 2016. p. 47.

22. Day MJ, Bilzer T, Mansell J, Wilcock B, Hall EJ, Jergens A, et al. International standards for the histopathological diagnosis of gastrointestinal inflammation in the dog and cat: a report from the World Small Animal Veterinary Association Gastrointestinal Standardization Group. J Comp Pathol. 2008;138:1-43.

23. Washabau RJ, Day MJ, Willard MD, Hall EJ, Jergens AE, Mansell J, et al. Endoscopic, biopsy, and histopathologic guidelines for the evaluation of gastrointestinal inflammation in companion animals. J Vet Intern Med. 2010;24:10-26.

24. Evans JA, Chandrasekhara V, Chathadi KV, Decker GA, Early DS, Fisher DA, et al. The role of endoscopy in the management of premalignant and malignant conditions of the stomach. Gastrointest Endosc. 2015;82:1-8.

25. Willard M, Mansell J. Correlating clinical activity and histopathologic assessment of gastrointestinal lesion severity: current challenges. Vet Clin North Am Small Anim Pract. 2011;41:457-63.

26. Tamura W, Fukami N. Early gastric cancer and dysplasia. Gastrointest Endosc Clin N Am. 2013:23:77-94.

27. Willard MD, Moore GE, Denton BD, Day MJ, Mansell J, Bilzer T, et al. Effect of tissue processing on assessment of endoscopic intestinal biopsies in dogs and cats. J Vet Intern Med. 2010;24:84-9.

28. Willard MD, Mansell J, Fosgate GT, Gualtieri M, Olivero D, Lecoindre P, et al. Effect of sample quality on the sensitivity of endoscopic biopsy for detecting gastric and duodenal lesions in dogs and cats. J Vet Intern Med. 2008:22:1084-9.

29. Subramanian V, Mannath J, Ragunath K, Hawkey CJ. Meta-analysis: the diagnostic yield of chromoendoscopy for detecting dysplasia in patients with colonic inflammatory bowel disease. Aliment Pharmacol Ther. 2011;33:304-12.

30. Rugge M, Fassan M, Pizzi M, Farinati F, Sturniolo GC, Plebani M, et al. Operative link for gastritis assessment vs operative link on intestinal metaplasia assessment. World J Gastroenterol. 2011;17:4596.

31. Jergens AE, Willard MD, Allenspach K. Maximizing the diagnostic utility of endoscopic biopsy in dogs and cats with gastrointestinal disease. Vet J. 2016;214:50-60.

\footnotetext{
Ready to submit your research? Choose BMC and benefit from:

- fast, convenient online submission

- thorough peer review by experienced researchers in your field

- rapid publication on acceptance

- support for research data, including large and complex data types

- gold Open Access which fosters wider collaboration and increased citations

- maximum visibility for your research: over $100 \mathrm{M}$ website views per year
}

At BMC, research is always in progress.

Learn more biomedcentral.com/submissions 